Turkish Journal of Geriatrics

DOI: 10.31086/tigeri.2020.145

2020; 23(1): 118-128

- Yaşar KESKIN ${ }^{1}$ iD

- Aysel GÜRCAN ATÇI

- Berna ÜRKMEZ ${ }^{3}$ D

- Yusuf Sinan AKGÜL ${ }^{4}$ (D)

- Nihal ÖZARAS 5 (D)

- Teoman AYDIN ${ }^{1}$ (D)

CORRESPONDANCE

Yaşar KESKIN

Bezmialem Vakif University, Department of

Physical Medicine and Rehabilitation, Istanbul,

TURKEY.

Phone: +905447876644

e-mail: ykeskin42@hotmail.com

Received: 21/10/2019

Accepted: 22/12/2019

${ }^{1}$ Bezmialem Vakif University, Department of Physical Medicine and Rehabilitation, Istanbul, TURKEY.

2 Baltalimanı Metin Sabancı Bone Diseases

Training And Research Hospital, Department of Physical Medicine and Rehabilitation, Istanbul, TURKEY.

${ }^{3}$ Haydarpasa Numune Education and Research Hospital, Department of Physical

Medicine and Rehabilitation, Istanbul,

TURKEY.

${ }^{4}$ Gebze Technical University, Department of Computer Engineering, Kocaeli, TURKEY.

${ }^{5}$ Avrasya Hospital, Department of Physical Medicine and Rehabilitation, Istanbul, TURKEY.

\section{EFFICACY OF A VIDEO-BASED PHYSICAL THERAPY AND REHABILITATION SYSTEM IN PATIENTS WITH POST-STROKE HEMIPLEGIA: A RANDOMIZED, CONTROLLED, PILOT STUDY}

\section{Abstract}

Introduction: The aim of this study was to evaluate the effects of video-based virtual reality exercises performed using the LeapMotion ${ }^{\circledast}$ device and Fizyosoft ${ }^{\circledast}$ software on the hand and upper extremity functions of patients with hemiplegia.

Materials and Method: The mean age of the patients in the study group was $63.6 \pm 7.1$ years and the duration of the disease was $13 \pm 10.74$ months. The mean age of the patients in the control group was $63.6 \pm 9.2$ years and the duration of the disease was $10 \pm 9.89$ months. This study was a randomized, controlled, singleblind study. The patients were randomly divided into two groups: an experimental group and a control group. Both groups were arranged to undergo a combination of a neurophysiological and a conventional exercise program and physical therapy. In addition, virtual reality training therapy was administered in the experimental group using the LeapMotion ${ }^{\circledR}$ device. The patients' range-of-motion (ROM) was measured using the Fizyosoft ${ }^{\oplus}$ software, which operates with the LeapMotion ${ }^{\circledR}$ device, and their upper extremity and general function status was evaluated using the Fugl-Meyer assessment of upper extremity motor impairment (FMA-UE) scale, the Brunnstrom recovery stage (BRS) scale, the Barthel Index (BI), and the Functional Ambulation Categories (FAC) scale at the beginning and end of treatment.

Results: Significant improvements were noted after treatment in the upper extremity range-of-motion values as well as in FMA-UE, BRS, BI, and FAC scores in the experimental and control groups. Additionally, there were significant differences in the ROM measurements of the hand and wrist joints between the groups. However, no significant differences in clinical test results were observed between the groups.

Conclusion: Virtual reality exercises have a positive effect on range-of-motion measurements in stroke patients. Studies with a larger number of participants are needed to demonstrate results more clearly using other scales.

Keywords: Hemiplegia; Stroke; Virtual reality; Rehabilitation. 


\section{INTRODUCTION}

Cerebrovascular diseases (stroke) are central nervous system disorders that occur as a result of the blockage or hemorrhage of vessels caused by disorders that develop in cerebrovascular structures and/or in the properties of the blood passing through these vessels (1). After stroke, patients with hemiplegia continue to have major movement- and function-related problems in $>50 \%$ of upper extremity functions (2). The limb affected by stroke is not used adequately; this tends to contribute to both reduced movement and dysfunction in daily living activities.

Neurodevelopmental treatment, proprioceptive neuromuscular facilitation, and Brunnstrom approach are among the conventional approaches for upper extremity rehabilitation following stroke, which aim to maximize upper extremity function (3).

With recent developments in computer systems, virtual reality (VR) applications are increasingly being used in the field of rehabilitation and have become well-established. Virtual/augmented reality applications enable users to move various objects in a virtual environment. The efficacy of this technology in treatment has been shown in various disorders, such as neurological diseases, diseases that cause acute/chronic pain, and fibromyalgia (4-6). However, it is not possible to adjust exercise parameters and inspect how accurately the patient performs the exercise in rehabilitation practices performed using game consoles. A unique system that allows these adjustments to be made, includes customized physical exercises, and uses depth sensors to follow developments and make necessary interventions, was developed and made available for use in the field of rehabilitation, and its effectiveness was demonstrated in some studies (7).

The aim of this study was to investigate the effect of VR applications developed specifically for the rehabilitation of patients with post-CVA hemiplegia on upper extremity functions and movements.

\section{MATERIALS and METHODS}

\section{Participants}

Twenty-four patients aged between 52 and 75 years who had a stroke for the first time and received inpatient treatment at the Physical Medicine and Rehabilitation Clinic between March 2016 and March 2018 were included in the study. All the recruited participants signed informed consent forms before participating in the study. The approval of the local Ethics Committee was obtained.

Patient with cognitive impairment, a Mini-Mental State Exam score $<23$, a disease other than stroke that would disrupt mobility, severe upper extremity spasticity (Modified Ashworth Scale score of 3 and above), a Brunnstrom motor staging score below 4 for the upper extremity, and patients who were unable to comprehend and perform instructions were excluded from the study.

Patients whose stroke diagnosis was verified by a neurologist through computerized tomography and/or magnetic resonance imaging techniques were randomized. However, the physician who conducted clinical evaluations in both groups were blinded to the patient group. Group $1 \mathrm{com}-$ prised patients who were given only a conventional rehabilitation program, and Group 2 comprised patients who were given both conventional and VR programs.

\section{Intervention}

\section{1- Conventional rehabilitation treatment} program

The treatment program of both the control and experimental group included physical therapy modalities and a combination of a neurophysiological and a conventional exercise program implemented 1 hour a day 5 days a week for a total of 6 weeks.

\section{2- Virtual reality treatment program}

VR exercises for the upper extremity (speed, difficulty, duration, etc.) were implemented for the experimental group using a developed software. In recent years, it has become possible to track 
hand movements in space using cameras with the LeapMotion ${ }^{\circledR}$ device. LeapMotion $\AA$ is a motion detection device that uses infrared optics and cameras to capture and monitor current motion without the use of conventional interface devices such as mouse and keyboards (Figure 1-a). Unlike other motion sensors, its distinctive feature is its sensitivity to a change of $0.01 \mathrm{~mm}$ in the movement of fingers as well as hands, with a capture rate of $300 \mathrm{~Hz}$. The LeapMotion $®$ controller has a surface area of $24 \mathrm{~cm} 2$ and detects the hand in a three-dimensional space with a field view of approximately $150^{\circ}$ (Figure $1 \mathrm{~b}-\mathrm{c}$ ). es multiple levels. It was designed for wrist flexion-extension, radial and ulnar deviations, forearm supination-pronation, and elbow flexion-extension movements (Figure 1e).

The LeapMotion $®$ device and monitor were set up in a quiet room with sound insulation where patients would not be affected by external factors. Before the patients started the session, they were instructed how to play the game. Apart from their routine rehabilitation programs, the patients performed VR exercises twice a week for 6 weeks under the supervision of a therapist.

Figure 1. a. View of hand on LeapMotion ${ }^{\circledR}$ device, b-c. Close-up view of hand in computer environment, d-e. Leapball and pong game in computer environment.
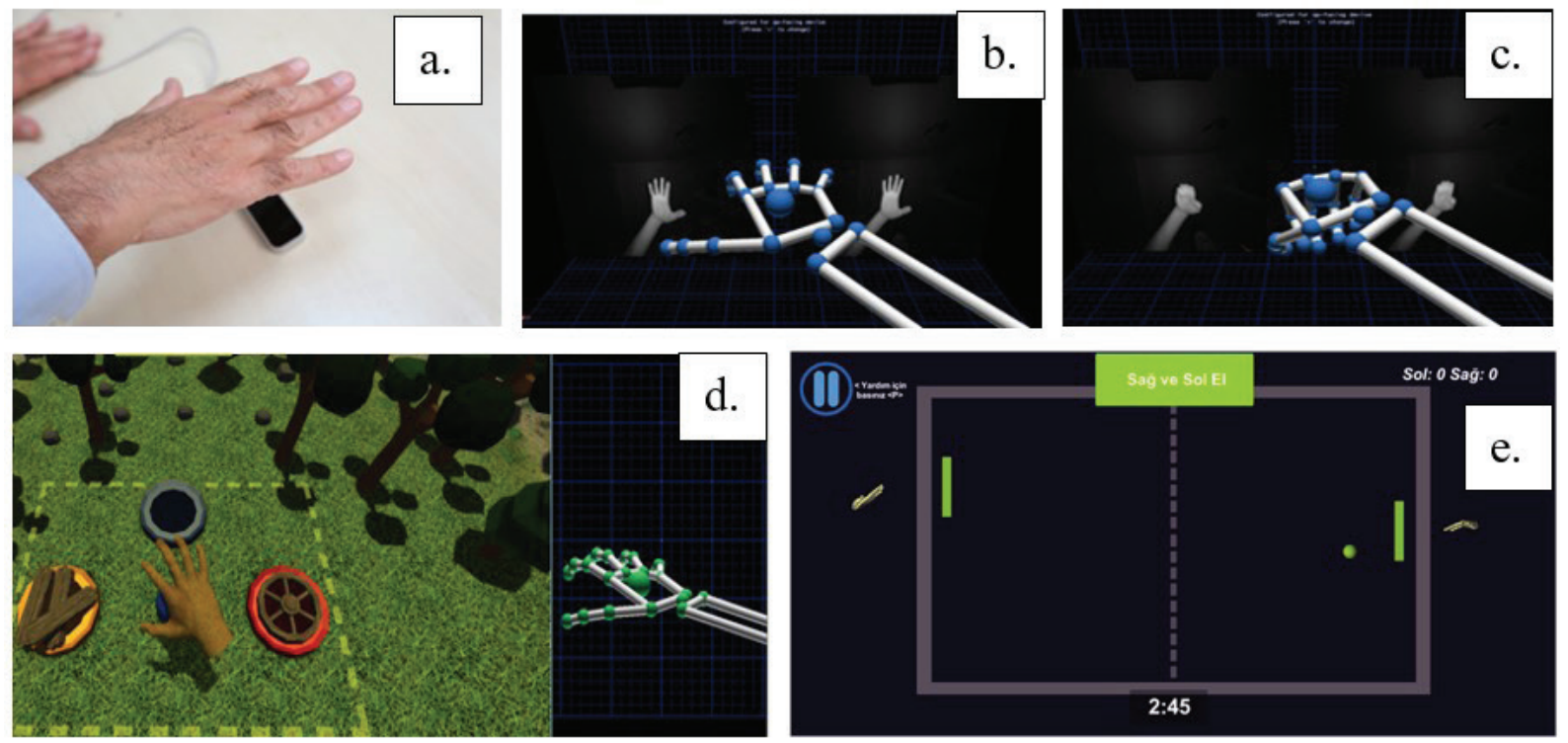

The LeapBall game application is a rehabilitation game developed for wrist flexion-extension and finger flexion-extension movements. It was primarily developed for hand grasp movements, and the game comprised multiple levels (Figure 1d).

The Pong game application was developed for wrist and elbow movements, and also compris-

\section{Assessment}

In this study, the upper extremity subscale of the Fugl-Meyer assessment of upper extremity (FMA-UE) scale was used to evaluate the motor function of the upper extremity. The FMA-UE scale is an index developed to assess sensorimotor recovery following stroke (8).

Evaluation of the neurophysiological recovery 
of the patients was conducted using the Brunnstrom recovery stages (BRS) developed by Brunnstrom for the hemiplegic upper extremity and hand (9). The Barthel Index (BI), for which a validity and reliability study was conducted on Turkish patients, was used in our study to evaluate the daily living activities of the patients (10). The ambulation status of all patients was evaluated using the Functional Ambulation Category (FAC) test (11).

FMA-UA, BRS, BI, and FAC scores of all patients were evaluated at the beginning of the treatment and 6 weeks after the beginning of treatment. In addition, the ROM results for joints were obtained using the LeapMotion $\AA$ device before treatment and 6 weeks after treatment.

Statistical analysis

The IBM SPSS Statistics 22 software was used for statistical analysis. In the evaluation of study data, normal distribution of the parameters was evaluated using the Shapiro-Wilk test and descriptive statistical methods (mean, standard deviation, and frequency) were used. Because the quantitative data did not show a normal distribution, the Mann-Whitney $U$ test was used as a non-parametric test for comparison between the two groups. The Wilcoxon signed-rank test was used for intragroup comparisons of parameters without normal distribution. The chi-square test or Fisher's Exact test were used to compare qualitative data. Parameters with a value of $p<0.05$ were considered statistically significant.

\section{RESULTS}

Twelve experimental patients with a mean age of $63.6 \pm 7.1$ years and 12 control patients with a mean age of $63.6 \pm 9.2$ years were included in the study. Demographic characteristics were similar between the groups $(p>0.05)$. The descriptive characteristics of the groups are shown in Table 1. In Group 1 , the patients' body mass index (BMI) was $29 \pm$ 4.87 , disease duration was $10 \pm 9.89$, and $92 \%$ of patients had an ischemic CVO. In Group 2, the pa-

Table 1. Demographic and clinical characteristics of the groups.

\begin{tabular}{|c|c|c|c|}
\hline & Group $1(n=12)$ & Group $2(n=12)$ & \multirow{2}{*}{$\mathrm{p}$} \\
\hline & Mean \pm SD & Mean \pm SD & \\
\hline Age, years (Mean \pm SD) & $63.6 \pm 7.1$ & $63.6 \pm 9.2$ & 0.750 \\
\hline Gender, female, n (\%) & $8(67)$ & $7(58)$ & 1.000 \\
\hline $\begin{array}{l}\text { Body mass index, } \mathrm{Kg} / \mathrm{m} 2 \text {, } \\
(\text { Mean } \pm \text { SD) }\end{array}$ & $29 \pm 4.87$ & $27 \pm 2.24$ & 0.299 \\
\hline $\begin{array}{l}\text { Time since stroke, months, } \\
(\text { Mean } \pm \text { SD) }\end{array}$ & $10 \pm 9.89$ & $13 \pm 10.74$ & 0.175 \\
\hline \multicolumn{4}{|l|}{ Affected extremity, n (\%) } \\
\hline Right/left & $4 / 8(33 / 67)$ & $5 / 7(42 / 58)$ & 1.000 \\
\hline Dominant & $12(100)$ & $12(100)$ & \\
\hline Comorbidities, n (\%) & & & 0.311 \\
\hline no & $0(0)$ & $2(17)$ & \\
\hline Hypertension & $5(42)$ & $5(42)$ & \\
\hline Diabetes mellitus & $7(58)$ & $5(42)$ & \\
\hline
\end{tabular}


tients' BMI was $27 \pm 2.24$, disease duration was 13 \pm 10.74 , and $75 \%$ of patients had an ischemic CVO.

We compared the post-treatment clinical data of the groups that did and did not receive VR treatment. When the patients were evaluated in terms of clinical examination results, no statistically significant difference was found between the two groups in FAS scale, FM upper extremity, FM lower extremity, and FM total scores, as well as their $\mathrm{BI}$ change and Brunnstrom upper extremity, Brunnstrom hand, and Brunnstrom lower extremity scores at the end of the 6th week after treatment.

Intragroup evaluation revealed that the improvements in the clinical scales resulted in statistically significant improvement in all clinical tests in both groups before treatment and at 6th week after treatment (Table 2).

Using this software, it is possible to measure ROMs of all joints from the elbow onward. In this respect, we compared the ROMs of the patients between the groups before and after treatment. There was no significant difference in the elbow and wrist ROMs of the groups following treatment.

A statistically significant intragroup improvement was noted before and after treatment in the plantar-flexion of the wrist in Group 1 and in the plantar-flexion of the wrist, ulnar deviation of the wrist, and elbow pronation in Group 2. Although there was improvement in other elbow and wrist ROMs, they were not statistically significant (Table 3).

In the analysis of finger movements of the groups, the ROMs in group 1 and Group 2 did not show a statistically significant difference. In the intragroup evaluation, it was observed that before and after treatment, improvement was greater in finger movements in the VR group, especially in DIP joint movements. In Group 1, significant improvement was not achieved only in the 1st PIP joint flexion and the 3rd DIP joint flexion; however, a statistically significant improvement was

Table 2. Baseline and post-intervention comparisons in outcome measures within and between the groups.

\begin{tabular}{|c|c|c|c|c|c|c|c|}
\hline & \multicolumn{2}{|c|}{ Group $1(n=12)$} & \multirow{3}{*}{$p^{*}$} & \multicolumn{2}{|c|}{ Group $1(n=12)$} & \multirow{3}{*}{$p^{\star}$} & \multirow{3}{*}{$\mathrm{p} \S$} \\
\hline & Baseline & $\begin{array}{r}\text { Post- } \\
\text { intervention }\end{array}$ & & Baseline & $\begin{array}{r}\text { Post- } \\
\text { intervention }\end{array}$ & & \\
\hline & Mean \pm SD & Mean \pm SD & & Mean \pm SD & Mean \pm SD & & \\
\hline FAC & $1.92 \pm 1.08$ & $3.75 \pm 1.14$ & 0.002 & $2.42 \pm 1.16$ & $3.83 \pm 0.94$ & 0.003 & 0.976 \\
\hline \multicolumn{8}{|c|}{ Fugl-Meyer Assessment UE } \\
\hline Total & $55.08 \pm 7.37$ & $62.08 \pm 3.23$ & 0.003 & $48.75 \pm 14.48$ & $53.58 \pm 13.13$ & 0.003 & 0.105 \\
\hline Wrist & $7.58 \pm 2.39$ & $9.17 \pm 1.53$ & 0.027 & $6.17 \pm 3.79$ & $7.25 \pm 3.47$ & 0.026 & 0.138 \\
\hline Hand & $9.92 \pm 2.23$ & $12.92 \pm 1.24$ & 0.006 & $9.92 \pm 4.14$ & $10.67 \pm 3.98$ & 0.105 & 0.227 \\
\hline Barthel index & $75.42 \pm 11.17$ & $88.33 \pm 14.03$ & 0.003 & $76.67 \pm 11.74$ & $88.75 \pm 7.42$ & 0.001 & 0.596 \\
\hline \multicolumn{8}{|c|}{ Brunnstrom Stages } \\
\hline UE & $5 \pm 0.74$ & $5.83 \pm 0.39$ & 0.004 & $5.25 \pm 0.62$ & $5.55 \pm 0.69$ & 0.046 & 0.261 \\
\hline Hand & $5.42 \pm 0.51$ & $6 \pm 0.38$ & 0.008 & $5.25 \pm 0.75$ & $5.55 \pm 0.82$ & 0.046 & 0.058 \\
\hline
\end{tabular}

Statistical analysis, *: Wilcoxon Signed Ranks test, §: Mann Whitney U test. FAC: Functional Ambulation Classification, UE: Upper Extremity. 
Table 3. Comparison of elbow and wrist range-of-motion before and after treatment of hemiplegic patients.

\begin{tabular}{|c|c|c|c|}
\hline & Group $1(n=12)$ & Group $2(n=12)$ & $n$ \\
\hline & Mean \pm SD & Mean \pm SD & 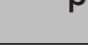 \\
\hline Elbow Pronation Pre & $76 \pm 26.35$ & $66 \pm 25.15$ & 0.562 \\
\hline Elbow Pronation Post & $78 \pm 23.58$ & $82 \pm 18.82$ & 0.603 \\
\hline $\mathrm{p}$ & 0.893 & 0.012 & \\
\hline Elbow Supination Pre & $27 \pm 27.71$ & $14 \pm 20.45$ & 0.198 \\
\hline Elbow Supination Post & $34 \pm 34.48$ & $32 \pm 33.52$ & 0.707 \\
\hline $\mathrm{p}$ & 0.386 & 0.173 & \\
\hline Wrist Flexion Pre & $11 \pm 11.83$ & $8 \pm 7.29$ & 0.536 \\
\hline Wrist Flexion Post & $32 \pm 23.65$ & $25 \pm 19.3$ & 0.339 \\
\hline $\mathrm{p}$ & 0.01 & 0.008 & \\
\hline Wrist Extension Pre & $51 \pm 16.06$ & $42 \pm 18.95$ & 0.797 \\
\hline Wrist Extension Post & $55 \pm 12.62$ & $53 \pm 14.3$ & 0.666 \\
\hline $\mathrm{p}$ & 0.508 & 0.11 & \\
\hline Wrist Ulnar deviation Pre & $20 \pm 8.26$ & $15 \pm 14.81$ & 0.166 \\
\hline Wrist Ulnar deviation Post & $23 \pm 8.98$ & $26 \pm 5.95$ & 0.774 \\
\hline $\mathrm{p}$ & 0.445 & 0.038 & \\
\hline Wrist Radial deviation Pre & $17 \pm 5.79$ & $18 \pm 2.94$ & 0.186 \\
\hline Wrist Radial deviation Post & $18 \pm 4.76$ & $17 \pm 6.98$ & 0.678 \\
\hline $\mathrm{p}$ & 0.463 & 0.779 & \\
\hline
\end{tabular}

achieved in other finger joints. In Group 2, a statistically significant improvement was observed in all other finger joints expect the 1st PIP, 2nd PIP, 3rd PIP, 4th PIP, and 5th PIP joint flexions (Table 4).

\section{DISCUSSION}

In this study, the effect of VR applications, in addition to conventional treatments, on upper extremity functions and movements in patients with hemiplegia was investigated. A significant improvement was observed in the pre- and post-treatment clinical evaluation tests in both the group in which
VR was implemented and the group that received only conventional rehabilitation therapy. While no significant difference was observed in elbow and wrist movements between the two groups, significant improvements were observed in some hand movements of the VR group.

It has been shown that because patients find VR to be more fun and exciting than other conventional rehabilitation programs, they put in more effort during VR-based treatment and therefore, more positive results are obtained due to positive reaction to this treatment and increased motivation (12). In addition, there are some arguments 
Table 4. Comparison of hand range-of-motion of patients before and after treatment.

\begin{tabular}{|c|c|c|c|}
\hline & Group $1(n=12)$ & Group $2(n=12)$ & \multirow{2}{*}{$\mathrm{p}$} \\
\hline & Mean \pm SD & Mean \pm SD & \\
\hline MCP Flexion Thumb Pre & $36 \pm 16.35$ & $34 \pm 16.38$ & 0.773 \\
\hline MCP Flexion Thumb Post & $50 \pm 9.33$ & $53 \pm 11.83$ & 0.395 \\
\hline $\mathrm{p}$ & 0.005 & 0.003 & \\
\hline MCP Flexion Index Pre & $40 \pm 13.71$ & $36 \pm 15.09$ & 0.299 \\
\hline MCP Flexion Index Post & $53 \pm 11.66$ & $51 \pm 10.18$ & 0.525 \\
\hline $\mathrm{p}$ & 0.005 & 0.005 & \\
\hline MCP Flexion Middle Pre & $38 \pm 13.86$ & $29 \pm 15$ & 0.083 \\
\hline MCP Flexion Middle Post & $51 \pm 12.61$ & $47 \pm 12.47$ & 0.326 \\
\hline $\mathrm{p}$ & 0.002 & 0.028 & \\
\hline MCP Flexion Ring Pre & $32 \pm 12.13$ & $28 \pm 12.95$ & 0.236 \\
\hline MCP Flexion Ring Post & $45 \pm 11.68$ & $39 \pm 17.42$ & 0.453 \\
\hline $\mathrm{p}$ & 0.002 & 0.099 & \\
\hline MCP Flexion Little Pre & $34 \pm 12.59$ & $28 \pm 16.46$ & 0.273 \\
\hline MCP Flexion Little Post & $51 \pm 17.36$ & $46 \pm 17.82$ & 0.564 \\
\hline $\mathrm{p}$ & 0.015 & 0.01 & \\
\hline PIP Flexion Thumb Pre & $38 \pm 16.84$ & $33 \pm 11.23$ & 0.954 \\
\hline PIP Flexion Thumb Post & $41 \pm 12.49$ & $35 \pm 8.45$ & 0.371 \\
\hline $\mathrm{p}$ & 0.347 & 0.754 & \\
\hline PIP Flexion Index Pre & $61 \pm 21.47$ & $49 \pm 18.85$ & 0.083 \\
\hline PIP Flexion Index Post & $73 \pm 14.75$ & $74 \pm 14.05$ & 1 \\
\hline $\mathrm{p}$ & 0.023 & 0.003 & \\
\hline PIP Flexion Middle Pre & $58 \pm 23.94$ & $40 \pm 22.45$ & 0.073 \\
\hline PIP Flexion Middle Post & $72 \pm 15.54$ & $70 \pm 20.07$ & 0.686 \\
\hline $\mathrm{p}$ & 0.023 & 0.019 & \\
\hline PIP Flexion Ring Pre & $50 \pm 24.48$ & $38 \pm 17.57$ & 0.225 \\
\hline PIP Flexion Ring Post & $64 \pm 23.85$ & $60 \pm 26$ & 0.795 \\
\hline $\mathrm{p}$ & 0.041 & 0.05 & \\
\hline PIP Flexion Little Pre & $50 \pm 25.91$ & $36 \pm 21.49$ & 0.204 \\
\hline PIP Flexion Little Post & $67 \pm 25.35$ & $65 \pm 23.01$ & 0.729 \\
\hline
\end{tabular}




\begin{tabular}{|c|c|c|c|}
\hline & Group $1(n=12)$ & Group $2(n=12)$ & \\
\hline & Mean \pm SD & Mean \pm SD & \\
\hline $\mathrm{p}$ & 0.019 & 0.008 & \\
\hline DIP Flexion Index Pre & $37 \pm 18.25$ & $56 \pm 16.8$ & 0.013 \\
\hline DIP Flexion Index Post & $58 \pm 21.74$ & $63 \pm 16.95$ & 0.544 \\
\hline $\mathrm{p}$ & 0.034 & 0.213 & \\
\hline DIP Flexion Middle Pre & $34 \pm 21.06$ & $55 \pm 19.44$ & 0.02 \\
\hline DIP Flexion Middle Post & $53 \pm 22.59$ & $58 \pm 17.34$ & 0.653 \\
\hline $\mathrm{p}$ & 0.062 & 0.594 & \\
\hline DIP Flexion Ring Pre & $33 \pm 21.13$ & $52 \pm 19.81$ & 0.035 \\
\hline DIP Flexion Ring Post & $54 \pm 19.25$ & $58 \pm 16.92$ & 0.477 \\
\hline $\mathrm{p}$ & 0.041 & 0.241 & \\
\hline DIP Flexion Little Pre & $34 \pm 23.27$ & $57 \pm 19.62$ & 0.011 \\
\hline DIP Flexion Little Post & $56 \pm 21.8$ & $65 \pm 14.36$ & 0.374 \\
\hline$p$ & 0.041 & 0.185 & \\
\hline
\end{tabular}

MCP: Metacarpophalangeal joints, PIP: proximal interphalangeal joints, DIP: distal interphalangeal joints

that the rehabilitation program implemented by mimicking the intended physical outcomes is more effective, and the VR program may be a solution for this treatment. In other studies conducted in this field, patients enjoyed performing in realistic environments and exhibited sufficient improvement in their physical abilities (13). In addition, activation in the corresponding neurological pathways of patients was demonstrated by functional magnetic resonance imaging during and after VR performed in a realistic setting (14).

However, there is little evidence in the literature showing the improvement of outcomes of rehabilitation in the physical environment. Although the authors have demonstrated that improvement can be achieved in rehabilitation results with the available realistic scenario and the rapidly implemented realistic application, research has not yet been able to show the main reason for the success of this program in the physical environment (15). This may be due to the fact that VR studies are difficult and expensive, and it is not possible to compare multiple VR programs with each other. Studies have shown that development has been achieved in upper extremity functions in patients diagnosed with hemiplegia through the game-based virtual reality application (16). It has been reported that the integration of an application used for gaming on Windows into the leapMotion $\AA$ controller has been effective for the fine motor skills of the hand functions of patients followed up for hemiplegia and contributed to the evaluation of fine motor skills (17). Losa et al. reported that an increase in hand skills was achieved using the leapMotion ${ }^{\circledR}$ controlled video games that they developed for adult patients diagnosed with subacute stroke (18).

With the virtual reality program that we implemented, the ROMs of all joints can be measured 
from the elbow onward, and the results can be monitored during patient follow-up. In addition, the exercises performed by patients at home can be recorded in the online system and followed up in a virtual environment.

In rehabilitation, the duration of the exercises and the number of repetitions of the movements are essential (19). The proposed virtual reality application provides patients with the ability to perform an unlimited number of repetitions while also ensuring the performance of a successful rehabilitation by drawing the patient's attention to the game environment on the screen. Patients will be able to be rehabilitated with a previously specified duration and number of repetitions in their homes in an environment where they feel comfortable and safe without having any fear. During the evaluation of results, patients' awareness of their clinical scores in the treatment process as well as discussion of the results and scores based on the knowledge and outlook of the patients' physiotherapist or physician will be more effective in motivating the patient (20).

While conventional treatment methods require minimal cognitive activity in the clinical environment during treatment, the patient may be unprepared for increased cognitive needs for the same performance outside of the clinic (such as listening and responding to someone while eating) $(21,22)$. VR may help in achieving the necessary cognitive attention with a set of adjusted scenarios, so that it can be ensured that instead of solely physical activity, patients' functions that require cognitive activity are also exercised. Researchers have already begun to write software designed to achieve this purpose. This software was developed for the recovery of impaired balance of patients against an unexpected stimulus $(21,22)$.

There are promising studies on the effectiveness of the VR program; however, studies on this treatment are still at the initial stages. Inability to obtain results from some studies may be due to the fact that researchers had limited time to develop and test their own VR programs. Nevertheless, the VR program is generally effective; in other words, it is a promising rehabilitation program that does not yield negative results. Whether or not the VR program would have been beneficial as an addition to conventional rehabilitation had been questioned until a few years ago; however, it has been recently included in rehabilitation research and practice. While there is no doubt regarding its benefit, it is recommended to perform a cost-benefit analysis before implementation $(15,23,24)$.

Our study also had some limitations. We found that there was no difference between some objective tests between the group administered and the group not administered VR treatment. This may be attributed to the use of this device, which is still under trial, in limited durations under hospital conditions. More accurate results may have been obtained if more patients were included in the study and if the patients were administered the treatment five times a week instead of only twice a week.

The strength of our study is that it is possible to use VR technology initially in hand and upper extremity rehabilitation and later in rehabilitation programs that involve the whole body in the subsequent stages due to the features of the VR technology and program. One of the most important advantages of the VR program is its applicability to all patients who present to rehabilitation clinics. Another limitation of our study is that it may cause anxiety and distress in patients using VR because they are not familiar with the virtual reality environment and because they feel they cannot. In order to overcome this situation, we have given patients a period of familiarization and tried to encourage patients. We believe that this VR program will be widely used considering the fact that every household today possesses a computer.

\section{CONCLUSION}

VR technologies used in rehabilitation have cer- 
tain advantages, such as the ability to implement the program at home, the elimination of time and space constraints, and the ability of the patient to use the time that needs to be allocated in a busy clinic in his/her own home or workplace. Rehabilitation programs customized for the patient should be developed using VR technologies together with conventional rehabilitation programs. Additionally, the target audience and exercise program of the

\section{REFERENCES}

1. Adams HP, Bendixen $\mathrm{BH}$, Kappelle LJ, et al. Classification of subtype of acute ischemic stroke. Definitions for use in a multicenter clinical trial. TOAST. Trial of Org 10172 in Acute Stroke Treatment. Stroke 1993;24(1):35-41. (PMID: 7678184).

2. Broeks JG, Lankhorst GJ, Rumping K, Prevo AJ. The long-term outcome of arm function after stroke: results of a follow-up study. Disabil Rehabil 1999;21(8):357364. ( PMID: 10503976).

3. Barclay-Goddard RE, Stevenson TJ, Poluha W, Thalman L. Mental practice for treating upper extremity deficits in individuals with hemiparesis after stroke. Cochrane Database Syst Rev 2011(5):Cd005950. (PMID: 21563146).

4. Llorens R, Noe E, Colomer C, Alcaniz M. Effectiveness, usability, and cost-benefit of a virtual reality-based telerehabilitation program for balance recovery after stroke: a randomized controlled trial. Arch Phys Med Rehabil 2015;96(3):418-425.e412. (PMID: 25448245).

5. Mirelman A, Maidan I, Deutsch JE. Virtual reality and motor imagery: promising tools for assessment and therapy in Parkinson's disease. Mov Disord 2013;28(11):1597-1608. (PMID: 24132848).

6. Keefe FJ, Huling DA, Coggins MJ, et al. Virtual reality for persistent pain: a new direction for behavioral pain management. Pain 2012;153(11):2163. (PMID: 22770840).

7. Ar I, Akgul YS. A computerized recognition system for the home-based physiotherapy exercises using an RGBD camera. IEEE Trans Neural Syst Rehabil Eng 2014;22(6):1160-1171. (PMID: 24860037).

8. Sullivan KJ, Tilson JK, Cen SY, et al. Fugl-Meyer assessment of sensorimotor function after stroke: standardized training procedure for clinical practice and clinical trials. Stroke 2011;42(2):427-432. (PMID: 21164120). virtual/augmented reality system that will be developed should be designed by experts, and studies should be planned with larger patient groups.

\section{FUNDING}

This work was supported by Scientific Research Projects Unit (Project No: 6.2015/5 n)

9. Naghdi S, Ansari NN, Mansouri K, Hasson S. A neurophysiological and clinical study of Brunnstrom recovery stages in the upper limb following stroke. Brain Inj 2010;24(11):1372-1378. (PMID: 20715900).

10. Kucukdeveci AA, Yavuzer G, Tennant A, Suldur N, Sonel B, Arasil T. Adaptation of the modified Barthel Index for use in physical medicine and rehabilitation in Turkey. Scand J Rehabil Med 2000;32(2):87-92. (PMID: 10853723)

11. Holden MK, Gill KM, Magliozzi MR. Gait assessment for neurologically impaired patients: standards for outcome assessment. Phys Ther 1986;66(10):15301539. (PMID: 3763704)

12. Merians AS, Poizner $H$, Boian $R$, Burdea $G$, Adamovich $\mathrm{S}$. Sensorimotor training in a virtual reality environment: does it improve functional recovery poststroke? Neurorehabil Neural Repair 2006;20(2):252-267. (PMID: 16679503).

13. Shema SR, Brozgol M, Dorfman $M$, et al. Clinical experience using a 5-week treadmill training program with virtual reality to enhance gait in an ambulatory physical therapy service. Phys Ther 2014;94(9):13191326. (PMID: 24786944).

14. Lucca LF. Virtual reality and motor rehabilitation of the upper limb after stroke: a generation of progress? J Rehabil Med 2009;41(12):1003-1006. (PMID: 19841832).

15. Subramanian SK, Lourenço CB, Chilingaryan G, Sveistrup H, Levin MF. Arm motor recovery using a virtual reality intervention in chronic stroke: randomized control trial. Neurorehabil Neural Repair 2013;27(1):13-23. (PMID: 22785001)

16. Shin J-H, Ryu H, Jang $\mathrm{SH}$. A task-specific interactive game-based virtual reality rehabilitation system for patients with stroke: a usability test and two clinical experiments. J Neuroeng Rehabil 2014;11(1):32. (PMID: 24597650). 
17. Khademi M, Mousavi Hondori H, McKenzie A, Dodakian L, Lopes CV, Cramer SC. Free-hand interaction with leap motion controller for stroke rehabilitation. Paper presented at: Proceedings of the extended abstracts of the 32nd annual ACM conference on Human factors in computing systems 2014. [Internet] Available from: https:// escholarship.org/content/qt4p831550/qt4p831550. pdf. (DOI:10.1145/2559206.2581203).

18. Iosa M, Morone G, Fusco A, et al. Leap motion controlled videogame-based therapy for rehabilitation of elderly patients with subacute stroke: a feasibility pilot study. Top Stroke Rehabil 2015;22(4):306-316. (PMID: 26258456).

19. Saleh S, Fluet G, Qiu Q, Merians A, Adamovich SV, Tunik E. Neural patterns of reorganization after intensive robot-assisted virtual reality therapy and repetitive task practice in patients with chronic stroke. Front Neurol 2017;8:452. (PMID: 28928708).

20. Seo NJ, Kumar JA, Hur P, Crocher V, Motawar B, Lakshminarayanan K. Usability evaluation of low-cost virtual reality hand and arm rehabilitation games. J Rehabil Res Dev 2016;53(3) (PMID: 27271199).
21. Heiden E, Lajoie Y. Games-based biofeedback training and the attentional demands of balance in older adults. Aging Clin Exp Res 2010. 22(5-6): p. 367373. (PMID: 20009499)

22. Yen $\mathrm{CY}$, Lin $\mathrm{KH}, \mathrm{Hu} \mathrm{MH}, \mathrm{Wu} \mathrm{RM}$, Lu TW, Lin $\mathrm{CH}$. Effects of virtual reality-augmented balance training on sensory organization and attentional demand for postural control in people with parkinson disease: a randomized controlled trial. Phys Ther 2011. 91(6): p. 862-874. (PMID: 21474638)

23. Holden MK, Dyar TA. Virtual Environment Training-A New Tool for Neurorehabilitation? Neurology Report 2002. 26(2): p. 62-71.

24. Holden MK, Dyar TA, L. Schwamm L, Bizzi E. Virtual-environment-based telerehabilitation in patients with stroke. Presence: Teleoperators \& Virtual Environments 2005. 14(2): p. 214-233. ( doi: 10.1162/1054746053967058). 